\title{
Wireless UAV Rotary Wing Communication with Ground Nodes Using Successive Convex Approximation and Energy Saving Mode
}

\author{
Dr. D. Sivaganesan \\ Professor, \\ Department of Computer Engineering \\ PSG Institute of Technology and Applied Research \\ Coimbatore, India \\ Email: sivaganesan@psgitech.ac.in
}

\begin{abstract}
Communication with several ground nodes (GNs) is enabled through wireless communications by means of a rotary-wing unmanned aerial vehicle (UAV). For every GN, the requirements of communication throughput is satisfied while minimizing the communication and propulsion related energy thereby reducing the total energy consumption of the UAV. The total completion time of the mission, the GN time allocation for communication as well as the trajectory of UAV are optimized jointly to formulate the problem of energy minimization. The UAV rotary wing model of power consumption for propulsion power in a closed-form is also derived. The complexity of the problem increases with the involvement of several variables infinitely over time and the non-convex variables, making it challenging to be solved optimally. The hovering location sets are visited by the UAV and at each location, communication is established with the corresponding GNs. This simplified design for fly-hover-communicate is established for tackling the underlying issue. Convex optimization techniques and travelling salesman problem with neighborhood (TSPN) are leveraged for optimization of the trajectory that connects the hovering locations and the duration of hovering by means of the proposed algorithm design. Communication of the UAV during operation is considered as a general scenario. Discretization of the problem to its equivalent is done in the proposed novel path discretization technique by means of optimized variables of finite number. Further, a successive convex approximation (SCA) scheme is applied for obtaining a solution that is locally optimal. A comparison of the proposed design is performed with the benchmark schemes and it is found that they are outperformed based on numerical results.
\end{abstract}

Keywords: Path discretization; trajectory optimization; energy-efficient communication; energy model; rotary-wing UAV; UAV communication;

\section{Introduction}

In locations that have insufficient terrestrial infrastructure, wireless coverage can be achieved by means of the promising technology of unmanned aerial platforms [1]. High altitude platforms (HAPs) techniques are considered mainly in early efforts for enabling ubiquitous coverage in remote or rural locations by deploying the UAVs at around $20 \mathrm{~km}$ altitude in the stratosphere [2]. In the recent days, there has been a growing interest in low altitude platforms (LAPs) wireless communication at a range of just a few kilometers over the ground level. Implementation of unmanned aerial vehicles (UAVs) and helikite kind of solutions is possible in LAPs along with several other schemes [3]. Wireless communication enabled UAVs are more advantageous compared to helikite, HAPs and other airborne solutions in general [4]. This is due to the features such as three dimensional (3D) airspace UAV movement with complete control and high network flexibility, swift and on-demand deployment, improved link quality with ground nodes (GNs) for communication channels with line-of sight (LoS) of shorter distance and so on [5]. Quick recovery of service in case of natural disasters, emergency response, collection of information for Internet of Things (IoTs) and dissemination of data, cellular base stations (BSs) temporary traffic offloading, communication safety for public and several such potential use cases are available for wireless communication enabled UAVs [6]. 


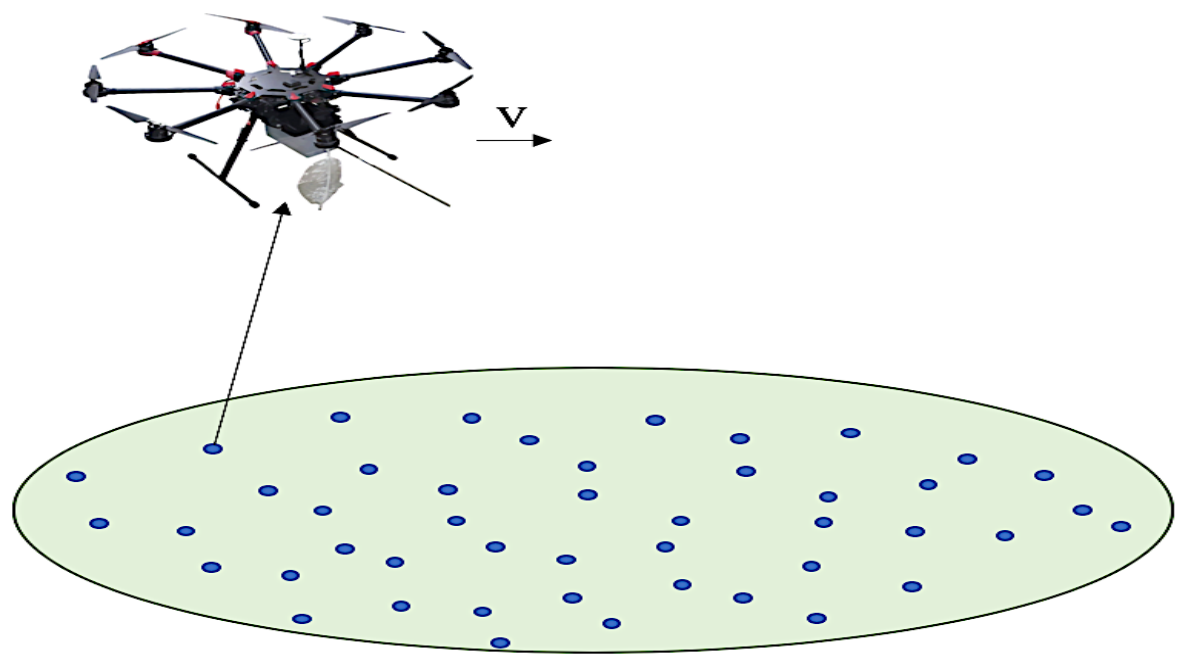

Figure 1: UAV rotary wing based wireless communication

Several researches are undertaken on wireless communication enabled UAVs that are generally classified into two major categories [7]. Deployment of UAVs as stationary (quasi) aerial base stations (BS) is the primary category [8]. New channel characteristics are enabled at higher altitudes in case of UAVs that are similar to the static terrestrial BSs of the conventional form. The probability of LoS between GNs and UAV increases with the increase in altitude of the UAV in particular [9]. Placement optimization of UAVs, cell association, spectrum sharing, performance analysis and other aspects of BSs enabled UAVs are analyzed thoroughly by understanding the unique characteristics of the channel [10]. The secondary category involves applications that deploy UAVs as access points (APs), relays and mobile BSs in which optimization of the performance of communication is to be established by the trajectory design. The GNs communication energy is saved and/or the overall efficiency of the spectrum is improved for communication between the UAV and the GNs associated to it as a data collector or mobile relay by flying closer to the GN [11]. UAV-enabled wireless power transfer, multi-UAV coordinated/cooperative communication and UAV-enabled data collection setups are enabled by extending the UAV enabled mobile relaying system proposed by optimization of UAV trajectory and enabling joint power allocation [12].

\section{System Model}

A wireless communication system is considered in which $\zeta=\{1, \ldots .$. ) is set of C GNs with which the communication of rotary-wing UAV is to be established. $\omega_{\mathrm{C}} \in \mathfrak{R}^{2 \times 1}$ is the horizontal location of the GN c $\in$ C. The constant altitude at which the UAV flies is denoted by A and the optimization of the altitude of UAV is exploited further. For the UAV to complete the mission, the total time necessary is denoted by a design variable $\tau_{\mathrm{t}}$. The projection of the trajectory of UAV on a horizontal plane is represented by $0 \leq \mathrm{t} \leq \tau_{\mathrm{t}}$ along with $\mathrm{q}(\mathrm{t}) \in \mathfrak{R}^{2 \times 1}$. The maximum speed of the UAV is denoted by $\mathrm{S}_{\max }\|\dot{q}(\mathrm{t})\| \leq \mathrm{S}_{\max }$ is the constraint. The distance between GN c and $\mathrm{UAV}$ at time instant $\mathrm{t} \in\left[0, \tau_{\mathrm{t}}\right]$, is given by

$$
d_{c}(t)=\sqrt{A^{2}+\left\|q(t)-\omega_{c}\right\|^{2}}, \mathrm{c} \in \zeta-\cdots---(1)
$$

The channel coefficient between the GN $c$ and UAV at time $t$ is denoted by $h_{c}(t)$. Decomposing $h_{c}(t)$, we get 
J. Sustain. Wireless Syst., vol. 02, no. 2, pp. 100-106, May 26, 2020

https://doi.org/10.36548/jsws.2020.2.006

$$
h_{c}(t)=\sqrt{\alpha_{c}(t)} \widetilde{h_{c}}(t)----(2)
$$

Where shadowing, path loss and other large-scale fading effects are represented by $\alpha_{c}(t)$ and a complex valued random variable $\widetilde{h_{c}}(t)$ with $\mathrm{E}\left[\left|\widetilde{h_{c}}(t)\right|^{2}\right]=1$ has a small-scale fading. Based on the probabilities of occurrence of LoS and non-LoS (NLoS) links, modeling of large scale attenuation for UAV-ground links as a random variable is done. $\alpha_{c}(t)$ can hence be written as

$$
\alpha_{c}(t)=\left\{\begin{array}{c}
\alpha_{0} d_{c}^{-\widetilde{\beta}}(t), \text { LoS link } \\
\zeta \alpha_{0} d_{c}^{-\widetilde{\beta}}(t), \text { NLoS link }
\end{array}\right.
$$

Here, the reference distance path loss is represented by $\alpha_{0}$, the path loss exponent is represented by $\tilde{\beta}$ the NLoS condition attenuation factor is represented by $\zeta<1$. The building height and density based statistic modeling and the propagation environment are the basis for LoS probability. At time t, the LoS probability between GN $c$ and UAV is modelled as a logistic function with an angle of elevation $\mathrm{P}_{\mathrm{c}}, \operatorname{LoS}(\mathrm{t})$ represented as follows:

$$
P_{c, L o S}(t)=\frac{1}{1+\operatorname{Kexp}\left(-D\left[\theta_{c}(t)-K\right]\right)^{\prime}}------(4)
$$

Here, the propagation environment decides the factors $\mathrm{K}$ and $\mathrm{D}$ and the elevation angle is represented by $\theta_{c}(t)=$ $\frac{180}{\pi} \operatorname{Sin}^{-1}\left(\frac{A}{d_{c}(t)}\right)$. The UAV trajectory $\mathrm{q}(\mathrm{t})$ decides the LoS probability $P_{c, L o S}(t)$.

\subsection{UAV Rotary-Wing Energy Consumption Model:}

Propulsion energy and energy related to communication are the two major components related to consumption of energy in the UAVs. Signal reception or radiation, signal processing, communication circuitry and several other factors affect the energy involved in communication. A power constant of $\mathrm{P}_{\mathrm{k}}$ watts is assumed for the power related to communication in the UAV. In order to support the movement of the UAV and keep it aloft, it is essential to have a propulsion energy consumption [13]. Acceleration and the flying speed of the UAV decides this propulsion energy consumption. Out of the total operational time of the UAV, a small duration is taken by the maneuvering scenarios such as acceleration or deceleration of the UAV and energy consumption due to it. These factors are ignored for enabling more tractable analysis and exposition of the scenario. Future work will cover the non-trivial power consumption model extension. By modeling the power consumption of propulsion for the UAV rotary wing that operates at a speed $\mathrm{S}$, we get

$$
P(S)=P_{0}\left(1+\frac{3 S^{2}}{V_{t i p}^{2}}\right)+P_{i}\left(\sqrt{1+\frac{S^{4}}{4 s_{0}^{4}}-\frac{S^{2}}{2 s_{0}^{2}}}\right)^{1 / 2}+\frac{1}{2} d_{0} \rho v A S^{3}
$$

Here, the constants $\mathrm{P}_{0}$ represents the blade profile power and $\mathrm{P}_{\mathrm{i}}$ represents the hovering status induced power. The rotor blade tip speed is given by $\mathrm{V}_{\text {tip }}$, the induced hovering velocity of the rotor is given by $\mathrm{v}_{0}$, drag ratio of fuselage is $\mathrm{d}_{0}$ and rotor solidity is $\mathrm{s}$. The rotor disc area is represented as A and the air density is $\rho$. Parasite power, induced power and profile power are the three major factors that affects the UAV rotary wing propulsion power consumption [14]. With the increase in V, there is a cubical and quadratic increase in the parasite power and blade profile power respectively. These factors aid in overcoming the fuselage drag and blades profile drag respectively. In order to overcome the blades induced drag, the induced power reduces with the decrease in $\mathrm{V}$. 
An easy, efficient as well as intuitive protocol, the fly-hover-communicate is used in this paper. For each GN, the optimized hovering locations $\mathrm{C}$ are visited successively by the UAV in this protocol. Communication is established at every corresponding location when the UAV hovers over the GN. The hovering locations connection path and optimal speed of flying, along with the C GNs hovering time allocation for communication and optimal hovering location identification is reduced [15]. An efficient solution is provided to this issue by the fly-hover-communicate protocol in which the time for completion of the mission is represented by $\mathrm{T}_{\mathrm{t}}$ does not decide the optimization variable count, instead it depends only on C. Binary flying status is used strictly for communication of the UAV in flight along with the sub-optimal protocol.

\section{Results}

In order to validate the design proposed, the numerical results are provided in this section. In case of small unmanned aircrafts whose flying heights are limited to 122 meters or 400 feet complying the FAA regulation, the altitude of $\mathrm{UAV}$ is set to $\mathrm{A}=100$ meters. $\widetilde{y_{0}}=53 \mathrm{~dB}$ is the obtained reference $\mathrm{SNR}$ and $\mathrm{P}=30 \mathrm{dBm}$ is the transmission power while the total bandwidth of communication is given by $\mathrm{B}=1 \mathrm{MHz}$. Excluding the propulsion power, the overall power consumption of the UAV is given by $\mathrm{P}_{k}=5 \mathrm{~W}$ for power related to UAV communication. The channel models based on special-free space as well as general probabilistic LoS are used for obtaining the results shown in figure 2, representing the convergence based energy minimization algorithm of the UAV.

$\tilde{\beta}=2.3, \varsigma=0.2, \mathrm{D}=0.6$ and $\mathrm{K}=10$ are the default parameters set for modeling the probabilistic LoS channel, unless stated otherwise. $\overline{P_{c, L o S}}$ which is the LoS probability that is regularized homogenously is set to a corresponding value with respect to the $90^{\circ}$ elevation angle. The parameters corresponding to the parameters of power consumption of UAV's propulsion are identified and analysed. $\mathrm{S}_{\max }=30 \mathrm{mps}$ is set as the maximum speed of flight of the UAV.q $\mathrm{q}_{\mathrm{I}}=[0,0]$ and $\mathrm{q}_{\mathrm{F}}=[800,800]$ is set as the initial as well as the final destination of the UAV. $\mathrm{C}=3 \mathrm{GN}$ is set up for experimental purpose. It is assumed that identical requirement of average throughput is considered for all GNs.

$$
\widetilde{Q_{c}}=\tilde{Q} \forall c \in C
$$


J. Sustain. Wireless Syst., vol. 02, no. 2, pp. 100-106, May 26, 2020

https://doi.org/10.36548/jsws.2020.2.006

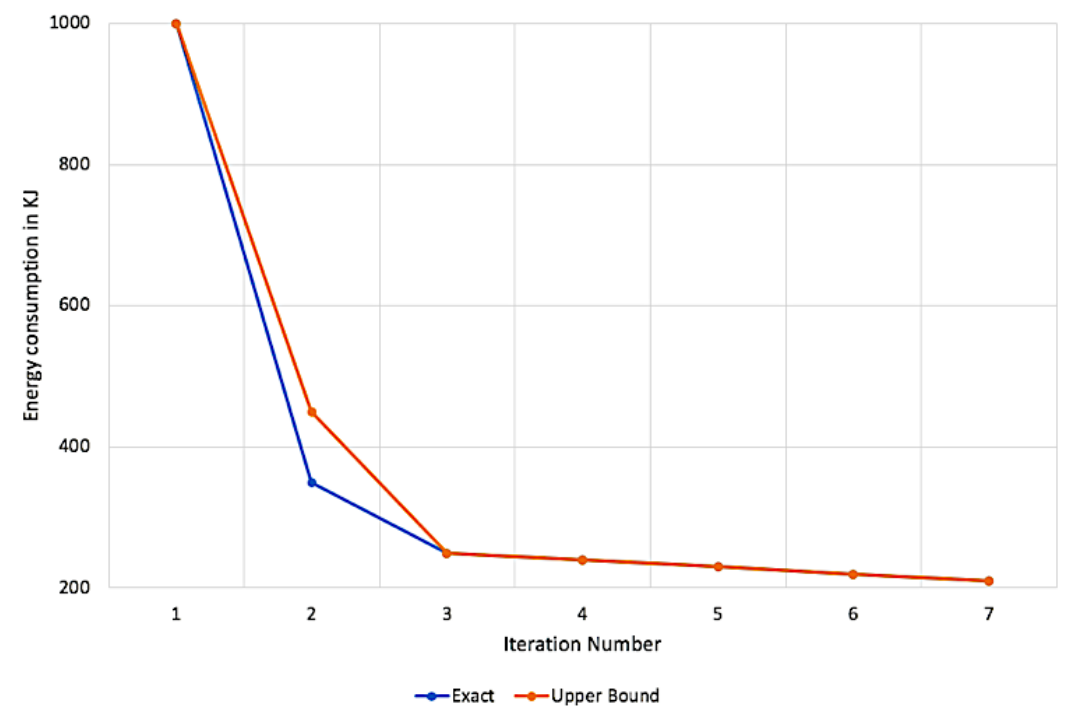

Figure 2: Convergence based Energy minimization algorithm for UAV

Finally, $\tilde{Q}$, the requirement of communication throughput versus time for completion of mission as well as the energy consumption of the UAV are compared. The continuous communication, optimized path, hovering above GNs and hovering at geometric center are the three main alternative benchmark schemes other than the mentioned three designs. Rather than optimization, the hovering locations are fixed at $\mathrm{C}$, which is the geometric center of each GN or the GNs in general. The fly-hover-communicate protocol extension is also used for this purpose. On solving linear programs over fixed paths, the allocation of an optimal UAV communication time and hovering time is obtained thereby enabling continuous communication.

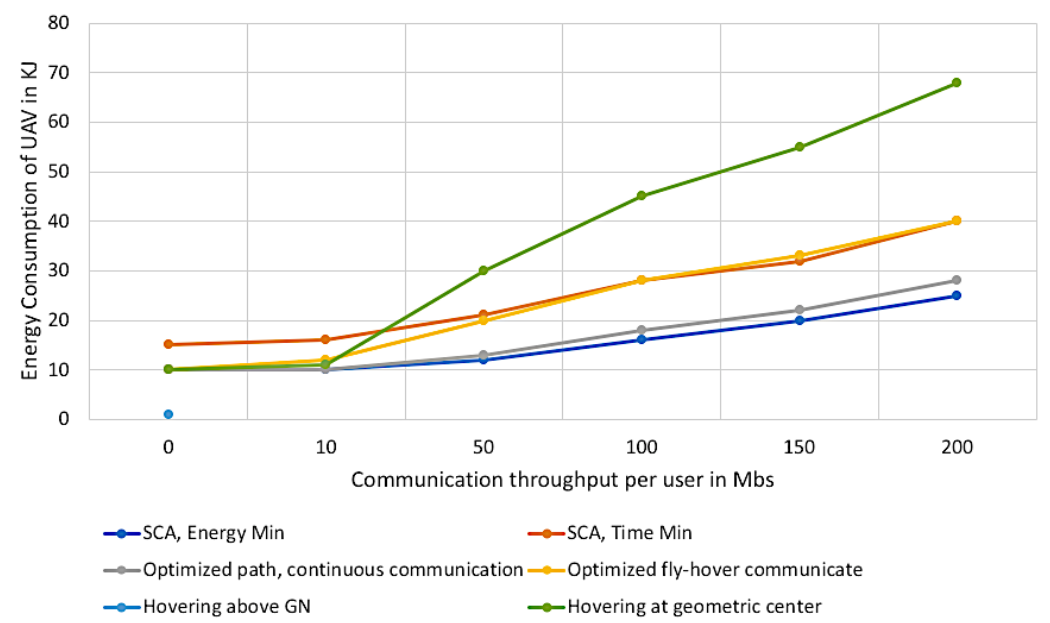


J. Sustain. Wireless Syst., vol. 02, no. 2, pp. 100-106, May 26, 2020

https://doi.org/10.36548/jsws.2020.2.006

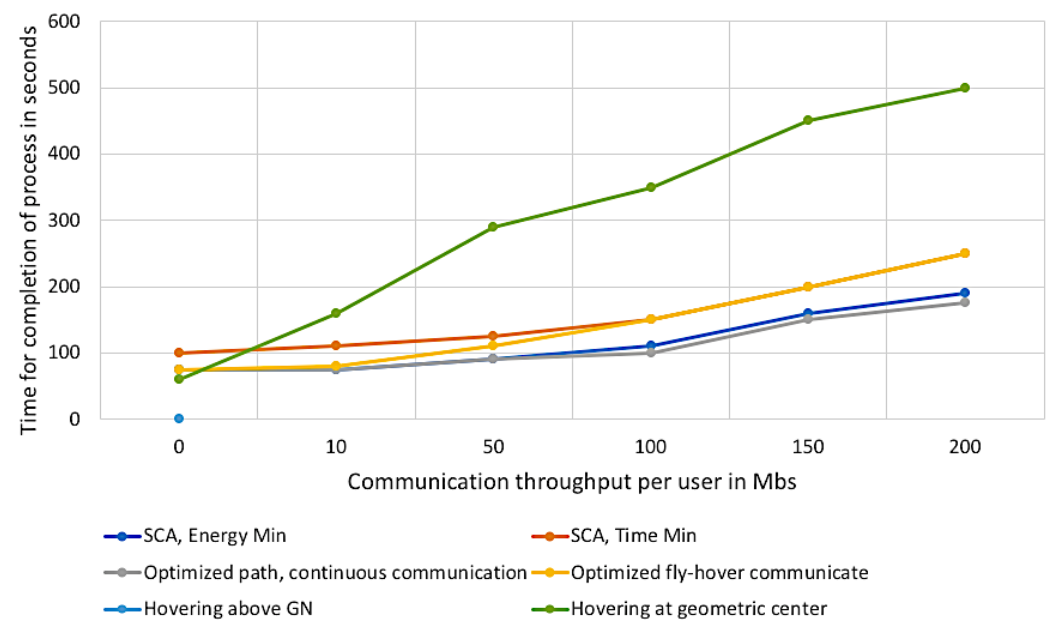

Figure 3: Mission completion time and Energy consumption vs throughput requirement

This scheme obtains a path that is identical to the fly-hover communicate scheme excluding the above mentioned differences. With the decrease in $\tilde{Q}$, the low requirements of throughput for hovering over GNs is outperformed by the hovering over the geometric center in terms of the essential mission completion time as well as the energy consumption as represented in Figure 3. Based on the requirements of communication, the hovering locations are optimized adaptively using the scheme of fly-hover communication that outperforms the traditional benchmark schemes. However, the fly-hover-communicate scheme is outperformed by the continuous communication of optimized path by enabling allocation of communication time and optimal hovering time thereby allowing continuous communication. Due to the improved balance between the maximization of communication rate and minimization of instantaneous power, energy saving is obtained by the proposed SCA algorithm. The design of time minimization also results in similar observations.

\section{Conclusion}

Communication of the rotary-wing UAVs in an energy efficient format is studied in this paper. An optimization problem for the UAV is formulated for reducing the total consumption of energy by deriving a model of propulsion power consumption for the rotary-wing UAV while the multiple GN throughput requirements for target communication are satisfied. An efficient solution is proposed based on a simple communication protocol of flyhover in which the speed and visiting order of the locations as well as the hovering duration and locations are optimized using convex optimization techniques and TSPN. Further, a general solution is proposed in which an SCA based novel path discretization approach is implemented for communication of UAVs in flight. For wireless communication systems of UAV rotary-ring, the benchmark solutions are outperformed by the proposed design according to numerical results.

\section{References}

[1] Wang, Z., Xu, W., Yang, D., \& Lin, J. (2019). Joint Trajectory Optimization and User Scheduling for Rotary-Wing UAV-Enabled Wireless Powered Communication Networks. IEEE Access, 7, 181369181380 .

[2] Zhan, C., \& Zeng, Y. (2019). Aerial-Ground Cost Tradeoff for Multi-UAV Enabled Data Collection in Wireless Sensor Networks. IEEE Transactions on Communications. 
[3] Zhan, C., \& Huang, R. (2020). Energy Efficient Adaptive Video Streaming with Rotary-Wing UAV. IEEE Transactions on Vehicular Technology.

[4] Li, S., Duo, B., Yuan, X., Liang, Y. C., \& Di Renzo, M. (2020). Reconfigurable intelligent surface assisted UAV communication: Joint trajectory design and passive beamforming. IEEE Wireless Communications Letters.

[5] Xu, Y., Xiao, L., Yang, D., Cuthbert, L., \& Wang, Y. (2019). Throughput maximization in UAV-enabled mobile relaying with multiple source nodes. Physical Communication, 33, 26-34.

[6] Li, R., Xiao, Y., Yang, P., Tang, W., Wu, M., \& Gao, Y. (2020). UAV-Aided Two-Way Relaying for Wireless Communications of Intelligent Robot Swarms. IEEE Access, 8, 56141-56150.

[7] Zhang, J., Zeng, Y., \& Zhang, R. (2020). Multi-Antenna UAV Data Harvesting: Joint Trajectory and Communication Optimization. Journal of Communications and Information Networks, 5(1), 86-99.

[8] Zeng, Y., Zhang, R., \& Lim, T. J. (2016). Throughput maximization for UAV-enabled mobile relaying systems. IEEE Transactions on Communications, 64(12), 4983-4996.

[9] Hou, J., Yang, Z., \& Shikh-Bahaei, M. (2018). Energy-Efficient Data Collection and Wireless Power Transfer Using A MIMO Full-Duplex UAV. arXiv preprint arXiv:1811.10134.

[10] Duo, B., Wu, Q., Yuan, X., \& Zhang, R. (2020). Energy Efficiency Maximization for Full-Duplex UAV Secrecy Communication. IEEE Transactions on Vehicular Technology, 69(4), 4590-4595.

[11] Wu, F., Yang, D., Xiao, L., \& Cuthbert, L. (2019). Energy consumption and completion time tradeoff in rotary-wing UAV enabled WPCN. IEEE Access, 7, 79617-79635.

[12] Yang, Z., Xu, W., \& Shikh-Bahaei, M. (2019). Energy Efficient UAV Communication with Energy Harvesting. IEEE Transactions on Vehicular Technology.

[13] Xiao, L., Xu, Y., Yang, D., \& Zeng, Y. (2019). Secrecy energy efficiency maximization for UAV-enabled mobile relaying. IEEE Transactions on Green Communications and Networking.

[14] Smys, S., \& Raj, J. S. (2019). A STOCHASTIC MOBILE DATA TRAFFIC MODEL FOR VEHICULAR AD HOC NETWORKS. Journal of Ubiquitous Computing and Communication Technologies (UCCT), 1(01), 55-63.

[15] Bhalaji, N. (2019). PERFORMANCE EVALUATION OF FLYING WIRELESS NETWORK WITH VANET ROUTING PROTOCOL. Journal of ISMAC, 1(01), 56-71.

\section{Authors Biography}

Dr. D. Sivaganesan is Professor in Department of Computer Engineering in PSG Institute of Technology and Applied Research Coimbatore, India his area of research includes Wireless Systems, Communication Networks, Internet of Things, Sustainable Computing, Computer Networks, Mobile Communication, Software Defined Wireless communication systems, Cyber Physical Systems, Green Data Centers, Cognitive principles and techniques. 